\title{
Open Total Talus Dislocation: A Case Report
}

\author{
E Tan, MBBS (IMU), HS Chua, MS Orth (UM), CL Ooi, MS Orth (UM), Zulkiflee O, MS Orth (USM) \\ Department of Orthopaedics, Hospital Pulau Pinang, George Town, Malaysia
}

\begin{abstract}
Total talus dislocation is a rare injury and is commonly accompanied by associated fractures. Common worrisome sequelae are infection, avascular necrosis and post-traumatic arthritis. We report here on a patient who sustained an open total talus dislocation with an ipsilateral medial malleolus fracture. Following early debridement, reduction and a combination of internal and external fixations, early recovery was good with no evidence of avascular necrosis.
\end{abstract}

\section{Key Words:}

Total talus dislocation, avascular necrosis, infection, outcome

\section{INTRODUCTION}

Total talus dislocation occurs when the talus is entirely detached and dissociated from all surrounding joints namely the tibiotalar, talonavicular and subtalar joints. It is a rare injury and commonly results from a high energy motor vehicle accident or fall from height. Total dislocation of the talus is usually associated with fractures of the malleoli or the talus itself. The mechanism of injury is thought to be due to excessive supination or pronation of the ankle joint resulting in either anterolateral or anteromedial dislocation with the former being more common ${ }^{1}$. In view of the nature of the injury, infection, avascular necrosis and post-traumatic arthritis are the most commonly anticipated major complications $^{2}$. Given the rarity of this injury, there are, to date, no established guidelines regarding treatment with a paucity of studies and literature regarding this type of injury. This case report describes a patient with an open talus dislocation associated with medial malleolus fracture who was treated with early surgical intervention and stable fixation.

\section{CASE REPORT}

A 39 years old gentleman was involved in a motor vehicle accident in which an oncoming vehicle collided with him causing him to fall sideways. He then lost consciousness and was unable to recall the exact mechanism of injury. Upon regaining consciousness, he complained of excruciating pain in the left ankle and was unable to bear weight. Examination of the left ankle revealed a total talus dislocation with the talus being exposed in its entirety from a contaminated wound at the anterolateral aspect of the ankle (Figure 1). Neurovascular integrity was not compromised. Radiographs confirmed a total talus dislocation with an associated displaced medial malleolus fracture (Figure 3).

Broad spectrum antibiotics were administered and the wound was irrigated upon arrival at the Emergency Department; further wound debridement and joint washout were performed under general anaesthesia. The talus was merely attached by a single strand of remaining ligament and all other talar soft tissue attachments were torn. There was bone loss at the antero-medial capsule aspect of the medial malleolus. The talus was reduced and screw fixation of the medial malleolus was performed. A cross ankle (tibiocalcaneal) external fixator was applied to maintain stability of the ankle joint (Figure 2). The wound was primarily closed with sutures. The patient was admitted for intravenous antibiotic therapy and wound care.

He was reviewed back in the clinic at follow-up intervals of two to four weeks. Repeated radiographs at six months posttrauma showed no evidence of avascular necrosis of the talus (Hawkin's sign was positive)(Figure 4) and a united medial malleolus fracture. All surgical and traumatic wounds healed uneventfully without evidence of superficial or deep seated infection. The external fixator was removed 12 weeks postoperatively and physiotherapy commenced The patient has since been able to bear weight on the affected ankle with minimal tolerable pain (pain score, 1 of 10) and is on long term follow up in anticipation of post-traumatic arthritis.

\section{DISCUSSION}

Total talus dislocation is a rare and unusual injury typically resulting from high energy trauma. Only a few case reports and small series have been published and there is no established treatment protocol for such injuries. There is also no concrete data regarding incidence rates for this injury. Kenwright and Taylor reported only two total talus dislocations out of fifty-eight major talar injuries $(3 \%)^{3}$.

Avascular necrosis is one of the most common complications that affect functional outcome in talar injuries and as such is a legitimate concern. Total extrusion involves disruption of vascular supply to the talus, predisposing to osteonecrosis. 


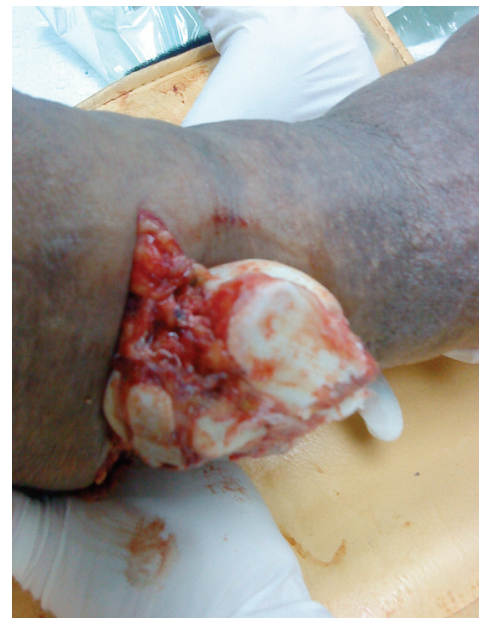

Fig. 1: Presentation of total talus dislocation with the talus exposed in its entirety from a contaminated wound at the anterolateral aspect of the ankle.

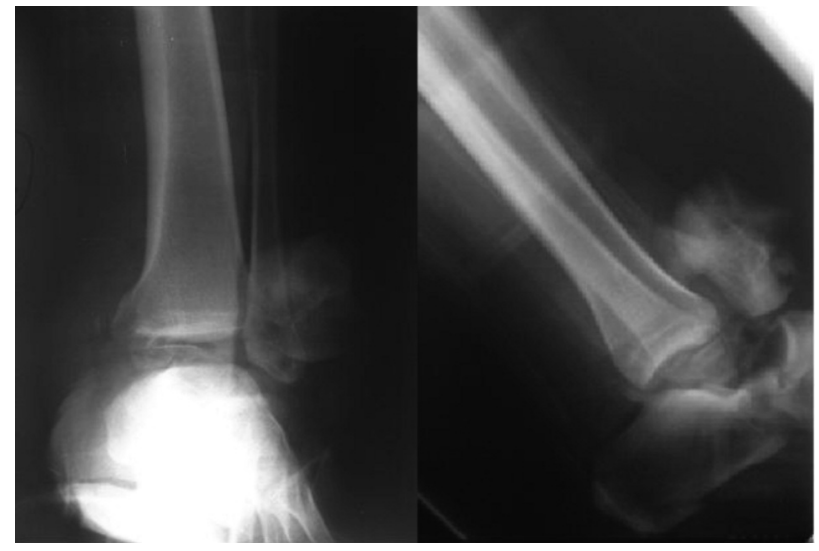

Fig. 3: Radiograph at time of injury showing a total talus dislocation and medial malleolus fracture.

The talus is known for its unique blood supply which poses a threat to its vascular integrity at time of injury. Approximately $60 \%$ of the talar surface is covered with cartilage which provides limited surface area for vascular perforation. The talar body is mainly supplied by the tarsal canal artery, which arises from the posterior tibial artery, and the deltoid artery. Branches of the dorsalis pedis artery and the tarsal sinus artery stem from the anterior tibial artery and supply the talar head. There is also a network of intraosseous arterial anastomoses with the most common being between the arteries entering the superior neck and the artery of the tarsal canal, both of which provide a rich blood supply to the talar body ${ }^{4}$. Additional capsular and ligamentous vessels adjoining the talus with the navicular, calcaneus and tibia also contribute to the blood supply of the talus. The talonavicular ligament has been shown to be a major nutrient supplier to the talus ${ }^{5}$. The risk of avascular necrosis is determined by disruption of the arterial supply and the the degree of soft tissue damage. Hence, revascularisation of the

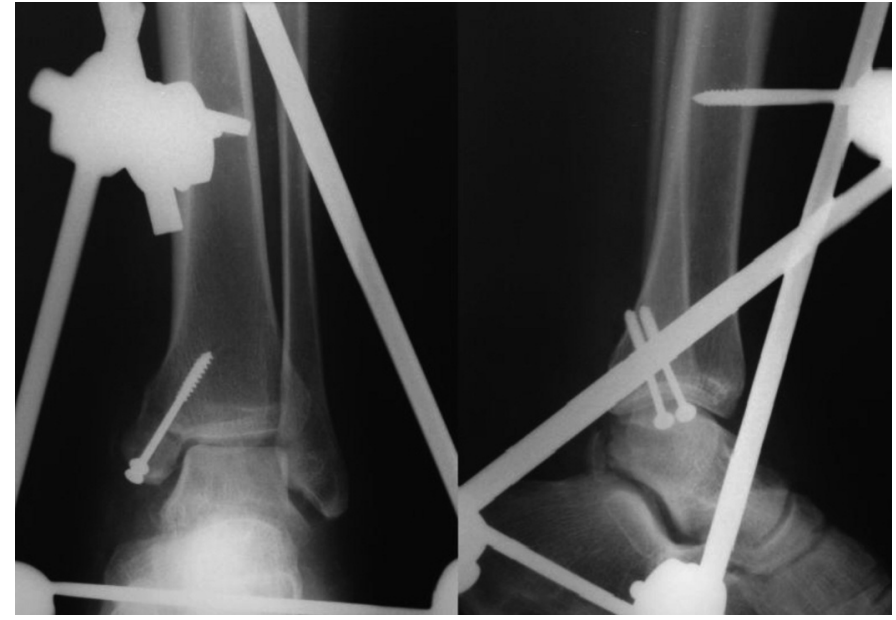

Fig. 2: Operative radiographs showing reduction of the talus and fixation of the medial malleolus.

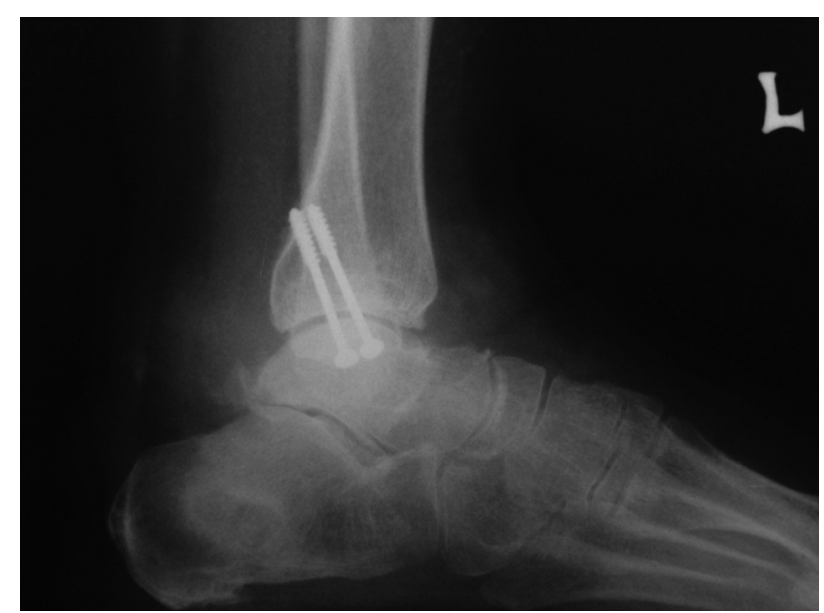

Fig. 4: Radiograph taken six months post-trauma showing no evidence of avascular necrosis.

talus occurs more easily when there are no fractures or when prompt soft tissue reattachment is performed to restore the vascular source.

Published evidence based treatment protocol for open injuries as such are scarce; treatment recommendations has evolved over the years from amputation to talectomy with tibiocalcaneal fusion and now recent case reviews suggest wound debridement, early reduction and fixation suffice to yield good clinical and functional outcomes ${ }^{2,5}$. Relocation of the talus is recommended provided the wound is relatively clean and the talus still attached, even if by a small strand of soft tissue ${ }^{5}$. Reduction and fixation of the talus in combination with complete wound debridement potentially successfully avoids infection, provides early revascularisation, and preserves the normal ankle anatomy. Further extensive surgical intervention such as talectomy and tibiocalcaneal fusion should be reserved as a salvage procedure. 
In the present case, wound irrigation and debridement was performed along with prompt initiation of intravenous antibiotics administration. The talus was then manually reduced and an external fixator was applied to allow wound care, soft tissue healing and immobilisation. Weight bearing was restricted for three months and upon follow up, a complete Hawkin's sign was noted indicating preservation of the talar blood supply. Based on previous reports, the management as mentioned in this case resulted in an environment close to the original biological state thereby encouraging early revascularisation. Avascular necrosis was also avoided as there was soft tissue, albeit minimal, still attached to the talus which promotes revascularisation ${ }^{5}$. However, development of post-traumatic osteoarthritis is still possible and may present years after initial injury. We recommend complete debridement, early talar reduction with sufficient soft tissue coverage and immobilisation in conjunction with weight bearing restrictions as key treatment points for prevention of common complications of such injuries.

\section{REFERENCES}

1. Leitner B. The mechanism of total dislocation of the talus. J Bone Joint Surg 1955; 37: 89-95

2. Hardy MA, Chuida S. Open Extrusion of the Talus: A case report. The Foot \& Ankle Journal. 2008; (12): 1

3. Kenwright J, Taylor RG. Major injuries of the talus. J Bone Joint Surg. 1970; 52(B): 36-48.

4. Bucholtz RW, Heckman JD, Court-Brown CM, Tornetta P, editors. Rockwood \& Green's Fractures in Adults; 6th Edition. 2006 Lippincott William and Wilkins. Chapter 54, Fractures of the Talus pp. 2256-7.

5. Newcomb WJ, Bray EA. Complete dislocation of the talus. J Bone Joint Surg. 1948; 30(A): 872-4. 